\title{
The importance of quality protein food in the treatment of fractures of tibia.
}

\author{
Schurov VA ${ }^{1^{*}}$ and Schurov IV ${ }^{2}$ \\ ${ }^{1}$ Department of Medical Sciences, Russian Ilizarov Scientific Center for Restorative Traumatology and Orthopedics, \\ Kurgan, Russia \\ ${ }^{2}$ Departments of Medical Sciences, Lomonosov Moscow State University, Russia
}

\begin{abstract}
The aim of the study is to analyze the reasons for the significant increase in terms of fixation of bone fragments when transferring patients to outpatient treatment. In 512 patients with closed fractures of the bones of the lower leg during Ilizarov's treatment under stationary conditions, the period of fixation of fragments was $59 \pm 0.4$ days, which is $42 \%$ less than in outpatient treatment (64 people). In both groups, the microscopic mobility of bone fragments was determined tensometrically in patients with limb functional loading. With the help of the SF-36 test, the quality of life was examined, and the state of autoregulation of the centers of the brain was assessed in terms of the range of changes in the rate of blood flow along the middle cerebral arteries in cases of muscle grasping. Discovered that the combination of medical and functional rehabilitation during the transition to outpatient mode with 3 -weeks of treatment in patients of mature age reduced quality of life and increases the duration of the period of fixation of the bone, impairs auto-regulation of cerebral blood flow in functional muscle test.
\end{abstract}

Keywords: Fracture of bones, Ilizarov method, Outpatient regimen, Quality of life, Autoregulation of cerebral blood flow.

Accepted on February 23, 2018

\section{Introduction}

In 1985 we paid attention to the significant increase in the duration of the fixation period in the treatment by Ilizarov patients with fractures of the limbs [1]. The actual duration of fixation was more recommended by author of the method of treatment by G. A. Ilizarov period in 54 days [2]. Exclude all possible reasons for this delay made it impossible to identify the determining factors in the deterioration of the quality of treatment. The subsequent period of "shock therapy population" associated with the socio-economic transformation of the society and a significant deterioration in the quality of life of the population suggests that the basis of deterioration in the quality of treatment can be prolonged deterioration in protein nutritional status of the population of the region $[3,4]$.

\section{Material and Methods}

512 analyzed case histories of patients with closed comminuted and spiral fractures of the tibia treated by Ilizarov method in the clinic of the scientific Center from 1970 to 2008 in age from 17 to 73 years $(44 \pm 1,2)$ with closed diaphyseal fractures of tibia bones. The second group (64) consisted of their peers with the same pathology in the dynamics of treatment in the years 2015-2017. All patients were determined mikromotion of fragments of the tibia under graduated, stepwise increasing to $10 \mathrm{kgf}$ axial loading of the limb. Checked the signal change of the strain gauge pre-stressed spring allowing an accuracy of 1 $\mu \mathrm{m}$ to determine the change in the distance between the spokes, leaving the bones above and below the zone of fracture [5].

Using ultrasonic Doppler installation "Angiodin-2 KM" (Russia) was determined by the linear blood flow velocity (FV) in middle cerebral arteries (MCA) at rest and again after you perform a functional test with the consistent work of the muscles of the wrist grab each side of the (compression elastic expander). Statistical processing of research results was conducted using analysis «Microsoft Exell-2010». The data contains the average values and standard error $(\mathrm{M} \pm \mathrm{M})$. When analyzing the dynamics of changes in indicators used methods of correlation and linear regression analysis (with the symbol c co-efficient of linear correlation $r$ or the index of determination R2). To assess the statistical significance of differences of results from two independent samples was used t-student test.

The result of the study Patients of the 1st group in the first weeks after osteosynthesis revealed a linear correlation relationship between indicators of micromotion of bone fragments $(\mathrm{L}, \mu \mathrm{m})$ and the duration of the fixation period ( $\mathrm{T}$, days): $\mathrm{T}=0.12 \times \mathrm{L}+51$, $36 ; \mathrm{R} 2=0.877$. The smaller mikrotverdosti determined by the accuracy of reduction and rigidity of fixation of bone fragments of the tibia, the faster was the fusion of bones. The average duration of the period of fixation of bone fragments was $59 \pm$ 1.4 day's duration of disablement $-97 \pm 3$ days. Consideration of the average functional load on the limb and the magnitude of micro motion of bone fragments in patients of the 1st group have spiral fractures after 3 weeks of treatment in patients of the $2^{\text {nd }}$ group at the end of the period of inpatient treatment did not reveal statistically significant differences. The quality of trauma care was high in both cases.

\section{Results and Discussion}

However, compared to 1985 , the time of fixation of bone fragments by 2005 increased when helical fractures by $45 \%$, and comminuted by $90 \%$ (Figure 1 ). The reason for increasing the duration of the fixation period could be the reduced reactivity of patients due to deterioration in the 
quality of life of the population of the region, in particular protein diet (Figure 2).

It is known that the age of patients also has an impact on the duration of treatment. Patients of the 1st group with increasing age the duration of the fixation period became longer (Figure 3 ).

Patients of the $2^{\text {nd }}$ group with increasing age also increased the duration of the period of fixation of bone fragments in the Ilizarov's device (Figure 4), which reached an average of $98 \pm$ 3.8 day. The nature of the curve changes after patients achieve retirement age.

Patients in this group under 30 years the increase of the micro motion associated with the regional resorption of bone fragments, occurred on the $19^{\text {th }}$ day of treatment, in patients over 50 years of age - after reaching the 40 days of treatment. The analysis of the dynamics of micro motion of bone fragments bones of the lower leg in the treatment of patients of the 1st group showed almost linear dependence on the time of fixation with the end in around 60 days (Figure 5). Patients of the $2^{\text {nd }}$ group after the transition to outpatient treatment with 15 to 60 days the magnitude of micro motion in most patients is not reduced, and only then (as the compaction of the regenerate) was their rapid decline. Term approximation of the rate of micro motion values of less than 25 microns, sufficient to end the fixation, it was increased by $90 \%$. However, in some patients of the $2^{\text {nd }}$ group of the first adult age dynamics of reduction of micro motion, functional loading of the limb and periods of fixation could not practically differ from that of patients of the 1st group.
If the duration of the fixation period began to exceed 67 days, there was a reduction in the duration of the subsequent period of functional rehabilitation (Figure 6). This fact confirms the position that in the process of fixing the bones of a limb is not only medical but also functional rehabilitation.

The indicator of quality of life, according to studies with the test SF-36 [1], in healthy examined with increasing age decreased from 81 to 74 points. Patients in the study conditions in the hospital in the first 10 days after injury figure were $61 \pm 2.8$ points. This relatively high rate in the first week of treatment caused by emotional upheaval associated with the restoration of the biomechanical integrity of the damaged bone in the second week with the restoration of the ability of patients not only to self, but also of locomotion. Through 60-80 days of treatment a measure of quality of life of patients was reduced to $40 \pm 4.7$ points $(t=3,93 ; p \leq 0.001)$ due to the reduction of modules that reflect the health status and social activity.

Indicator of adequacy of reaction of functional systems to increase motor activity is the state of auto regulation of the blood supply to the somatosensory centers of the brain.

In healthy people throughout life, including in terms of agerelated decline in absolute values of cerebral blood flow, must maintain an optimal level of auto regulation, which was judged by the magnitude of the changes of FV in MCA in functional muscle samples. This scope is in adults at hospital level, equal to $21 \% \pm 1.6$ [3]. With increasing age, indicator of the scope of change started increased steadily (Figure 7). Such a deviation

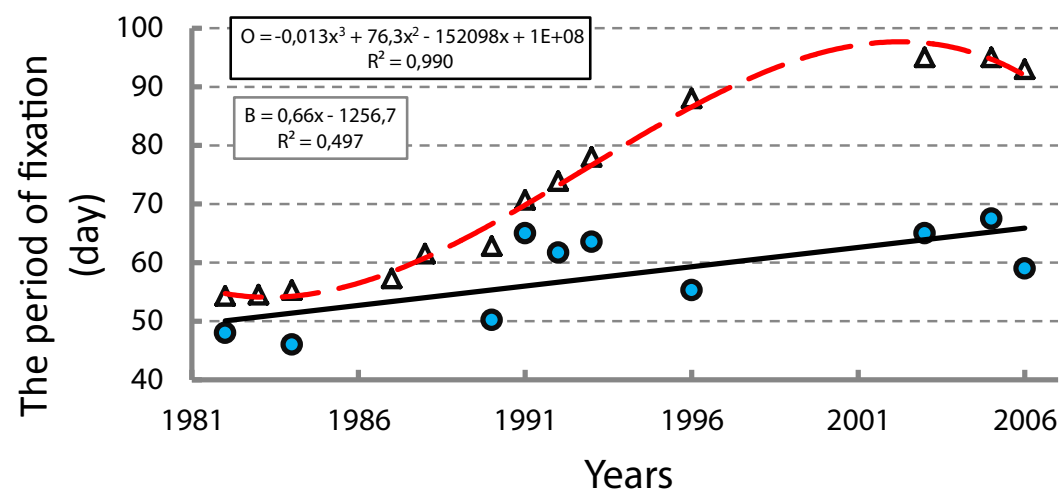

Figure 1. Dynamics of the duration of the period of fixation of bone fragments in patients with comminuted and spiral fractures of tibia bones.

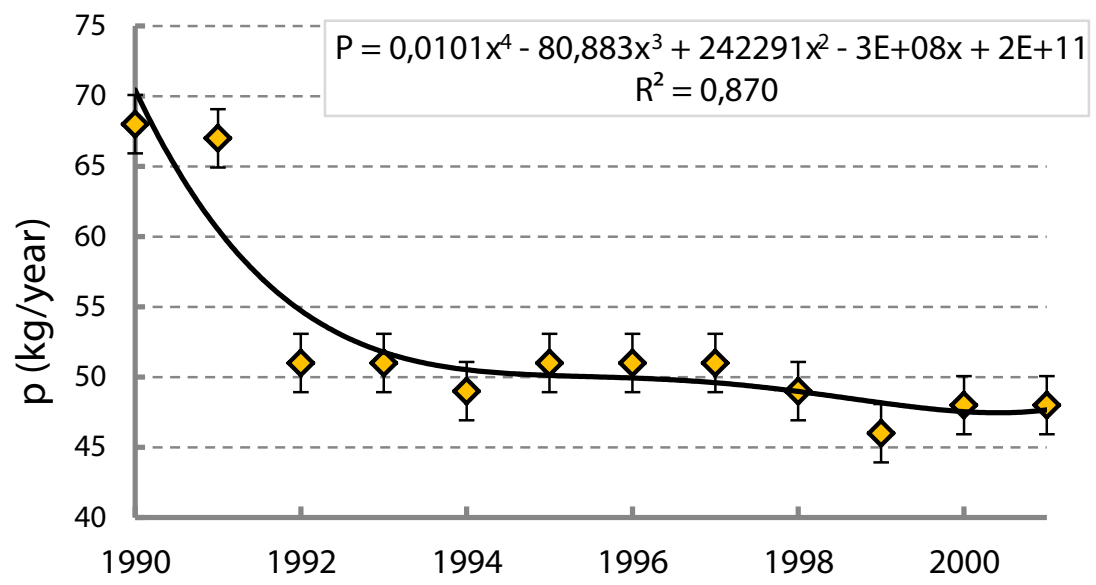

Figure 2. Dynamics of annual per capita meat consumption by the population of the Kurgan region. 


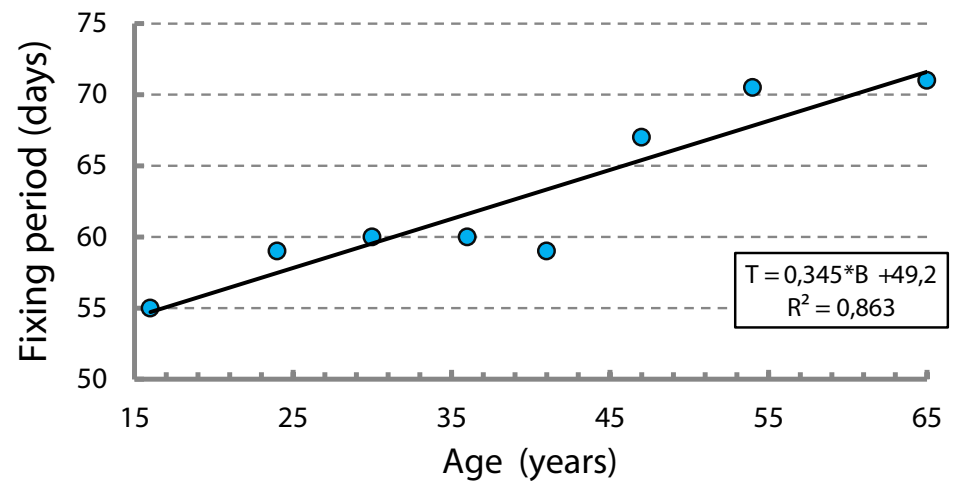

Figure 3. Age dynamics of duration of fixation of bone fragments in patients with injuries of the tibia of the 1 group.

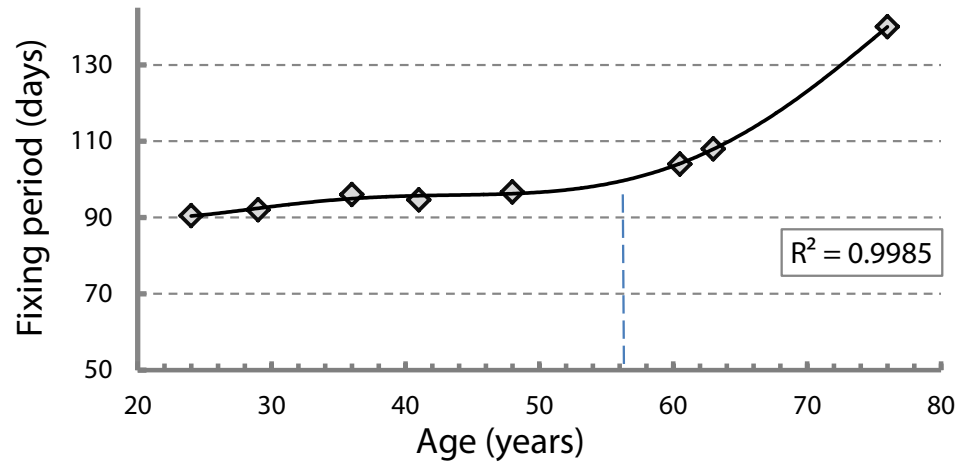

Figure 4. Age dynamics of duration of fixation of bone fragments patients with injuries of the lower leg of the 2nd group.

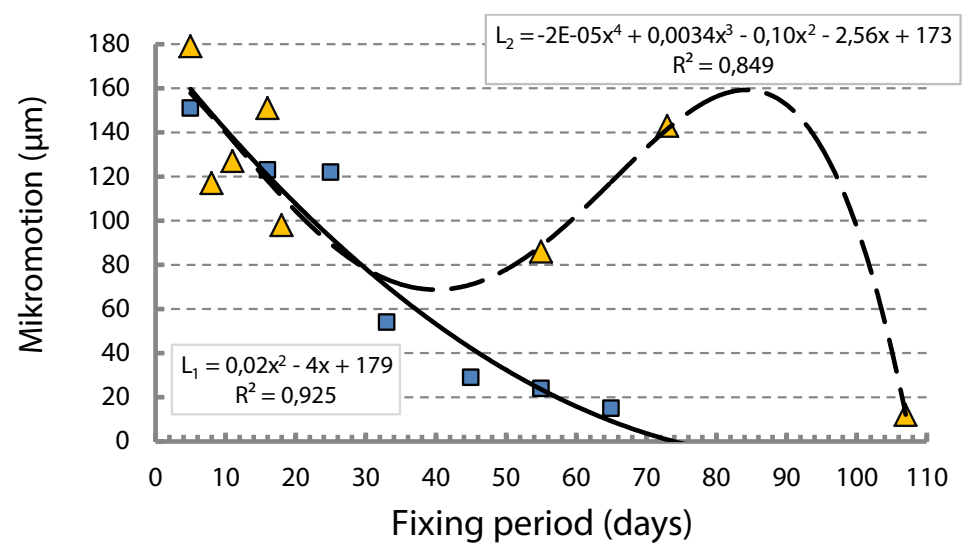

Figure 5. Dynamics of micromotion of bone fragments in the treatment of patients with a fracture of the tibia in the treatment in the hospital (1) and in the clinic (2).

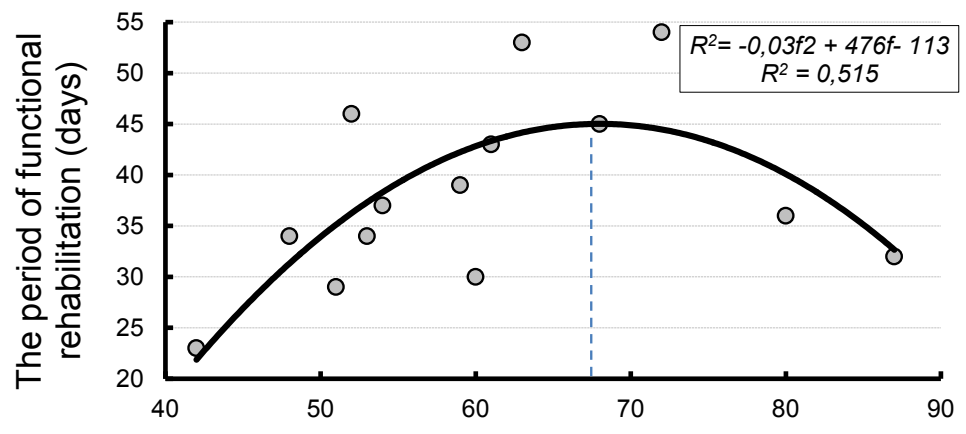

Fixing period (days)

Figure 6. The relationship between the duration of periods of fixation and functional rehabilitation of patients. 


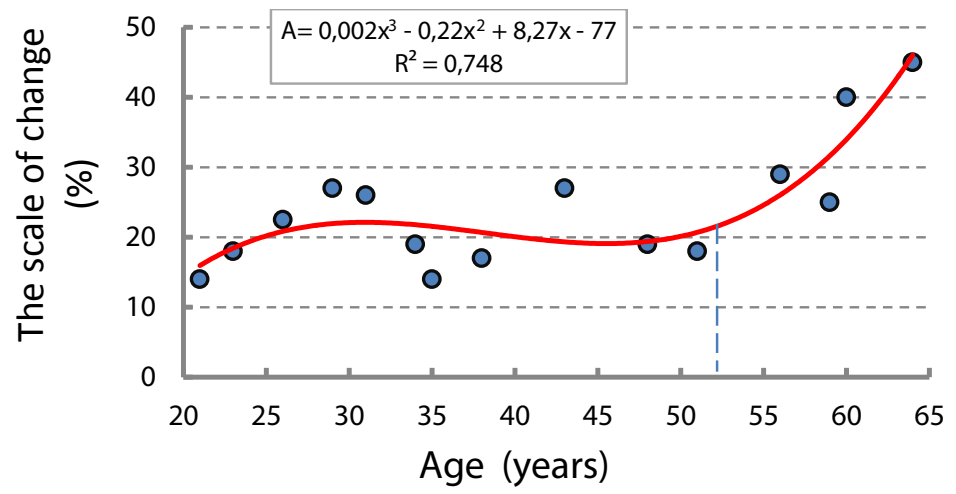

Figure 7. Age changes in the magnitude of changes in the rate of cerebral blood flow during functional test in patients with fracture of the tibia of the 2nd group.

from the norm is an unfavorable indication of a violation of auto regulation of the blood supply to the brain; it is an inadequate response to additional physical activity.

\section{Conclusion}

Therefore, in the face of declining age-related reactivity of the patients in the shortage of protein food, their treatment by the Ilizarov method in the outpatient setting leads to a violation of the regulatory functions of the centers of the brain, accompanied by a delay of reparative regeneration and prolong the period of fixation of bone fragments.

\section{References}

1. Hopman WM, Towheed T, Anastassiades T, et al. Canadian normative data for the SF-36 Health Survey. Canadian Medical Association or its Licensors. 2000;163:265-271.
2. Ilizarov GA, Devyatov AA. Possibilities of transosseous osteosynthesis in the treatment of fractures of bones In: Treatment of fractures and their consequences method of transosseous osteosynthesis. 1979:4-8.

3. Schurov VA. Analysis of the age of speed reduction of cerebral blood flow. Regional Circulation and Microcirculation. 2016:54-59.

4. Schurov VA. Power quality and reactivity of orthopedic and trauma patients, obstetric patients and newborns. Balanced diet, nutritional supplements and bio stimulants. 2016:84-86.

5. Schurov VA. Evaluation of the biomechanical and functional maturity of the bone regenerate. Human Physiology. 2018;4:102-107.

\section{*Correspondence to:}

Schurov VA

Department of Medical Sciences

Russian Ilizarov Scientific Center for Restorative Traumatology and Orthopedics

Kurgan

Russia

Tel: +7 (3522) 454758

E-mail: shchurovland@mail.ru 